\title{
Stars and singularities: Stellar phenomena near a massive black hole
}

\author{
Tal Alexander \\ To appear in \\ The Galactic Black Hole \\ H. Falcke \& F. W. Hehl, eds. \\ Institute of Physics 2002
}




\section{Contents}

Contents

1 Stars and singularities: Stellar phenomena near a massive $\begin{array}{ll}\text { black hole } & 1\end{array}$

1.1 Introduction 1

$\begin{array}{lll}1.2 & \text { Stellar dynamics near a black hole } & 3\end{array}$

$1.2 .1 \quad$ Physical scales $\quad 4$

$\begin{array}{lll}1.2 .2 & \text { A relaxed stellar system around a } \mathrm{MBH} & 6\end{array}$

1.3 The stellar collider in the Galactic Center 9

1.3.1 The case for a dense stellar cusp in the Galactic Center 9

12.3 .2 Tidal spin-up 12

$\begin{array}{lll}1.3 .3 & \text { Tidal scattering } & 13\end{array}$

1.4 The gravitational telescope in the Galactic Center 17

$\begin{array}{lll}1.4 .1 & \text { Gravitational lensing by a point mass } & 18\end{array}$

$\begin{array}{lll}1.4 .2 & \text { Pinpointing the MBH with lensed images } & 20\end{array}$

\begin{tabular}{|lll}
1.4 .3 & The detection of gravitational lensing & 24
\end{tabular}

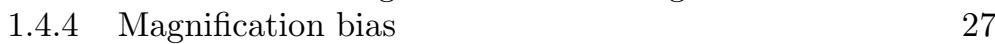

1.4.5 Beyond the point mass lens approximation $\quad 29$

1.5 Summary 30 


\title{
Chapter 1
}

\section{Stars and singularities: Stellar phenomena near a massive black hole}

\author{
Tal Alexander \\ The Weizmann Institute of Science
}

\section{$1.1 \quad$ Introduction}

Isolated black holes are simple objects, characterized by three numbers only: mass, angular momentum and charge. The complexity arises from the interaction with their surroundings, which results in a wealth of physical phenomena. This chapter will focus on the interaction of the central $\sim$ $3 \times 10^{6} M_{\odot}$ massive black hole $(\mathrm{MBH})$ in the Galactic Center (Genzel et al. 2000) with the stars very close to it. It will discuss processes for which there is already some observational evidence, as well as processes that are suggested by theory and may yet be discovered by future observations of the MBH in the Galactic Center or of central MBHs in other galaxies.

The MBH environment is unique because in addition to the gravitational singularity, there are three other "effective singularities" that are associated with the MBH. (1) A stellar density singularity. This is predicted to occur in most scenarios for the evolution of a stellar system around a MBH (e.g. Bahcall \& Wolf 1976, 1977; Young 1980). A density distribution that formally diverges at the origin is called a cusp. In practice, infinite density is not reached. Stars cannot exist closer than the event horizon, and in fact they are destroyed well before that point either by collisions or by the MBH tidal field. (2) A velocity singularity. Close to the MBH the velocity field is Keplerian and so formally diverges as $r^{-1 / 2}$. The velocity cusp is also limited in practice by the absence of stars arbitrarily close to the MBH. (3) An optical singularity. Any mass bends light and amplifies the flux of background sources. Behind the MBH (or any other sufficiently compact mass) there is a small region (a caustic) where the amplification 
formally diverges to infinity. This divergence is truncated by the finite size of the source.

The discussion will focus on the consequences of these singularities on stars near the MBH, where the term "near" is defined here to mean the region where stars can exist (i.e. beyond the tidal radius) but where the potential is completely dominated by the MBH. For the Galactic Center, the event horizon is much smaller than the tidal radius (for a solar type star) and so General Relativistic effects can be neglected to first approximation.

There are several reasons to study stars near the MBH. First, unlike gas, whose dynamics can be influenced by non-gravitational forces such as thermal pressure, radiation pressure and magnetic fields, stars are clean gravity probes. The properties of stars are well known from other environments, and their observed luminosity and spectrum can be translated into mass and maximal age. Both these quantities are very important for understanding the dynamics of the system. In particular, processes that operate on time-scales much longer than the maximal stellar age cannot be relevant for the star. Second, stars very near the MBH are connected to the growth of the MBH through tidal disruption, mass loss from stellar winds and from stellar collisions. Third, the region near the MBH can provide a unique laboratory for studying stellar phenomena under extreme conditions: high density, velocity and strong tidal fields.

Presently, infrared spectroscopy is possible for the brighter, well separated stars in the field. Spectroscopy indicates that the stellar population is a mix of old (red) stars and young (blue) stars (see review by Genzel, Hollenbach \& Townes 1994). The old red giants seen near the MBH in the Galactic Center are in the mass range $\sim 1-8 M_{\odot}$ and are older than 1 Gyr. The faintest observable young blue giants may be main sequence B1 or O9 stars with masses of $\sim 20 M_{\odot}$ and main-sequence lifetimes $<5$ Myr. The brightest young stars, the "He stars", are Wolf Rayet-like stars with masses of $>20 M_{\odot}$ and lifetimes of $<10 \mathrm{Myr}$. The blue stars are too young to have relaxed dynamically, and their orbits (position, velocity) still reflect the initial conditions of their formation (e.g. the young blue emission line giants are observed to counter rotate relative to the galactic rotation).

All the stars in the inner $0.02 \mathrm{pc}$ around $\mathrm{SgrA}^{\star}$ are faint, and have blue featureless spectra, which are typical of young stars. The fact that there are only seemingly young stars very close to the $\mathrm{MBH}$, while there is a mixture of young and old stars farther out raises a "Nature vs Nurture" question. Is this an essentially random variation in the stellar population, which can be explained in terms of normal star formation processes ("Nature"), or is this a result some systematic effects of the unique extreme environment very near the MBH ("Nurture")? It is interesting to note that a cluster of blue stars exists also around the $\sim 3 \times 10^{7} M_{\odot} \mathrm{MBH}$ in the galaxy M31 (Lauer et al. 1998).

If these stars are indeed the products of their environment, then there 
are two options to consider. First, this could be the result of an unusual mechanism of star formation (Morris 1993), in which case the stars are indeed young and dynamically unrelaxed, and so do not convey direct information on the dynamical processes in near the MBH. Second, this could be a results of unusual stellar evolution, so that the stars only appear young, but are in fact old and dynamically relaxed. This chapter will focus on the second possibility $(\S 1.3)$. However, before we discuss possible mechanisms for modifying stellar evolution, it is useful to review some results from stellar dynamics theory.

\subsection{Stellar dynamics near a black hole}

The stellar dynamical term "stellar collision" is not limited to the case of actual physical contact between stars, but refers to any gravitational interaction where the stars exchange momentum or energy. The dynamical processes in a gravitating stellar system can be summarized by considering stellar collisions as function of their distance scale. The reader is referred to Binney \& Tremaine (1987) for a detailed overview.

On the largest scale, the motion of the star is determined by the sum of interactions with all the other stars, that is, by the smooth gravitational potential of the system. Two-body interactions occur on a shorter length scale, when two stars approach each other to the point where their mutual interaction dominates over that of the smoothed potential. Two-body interactions randomize the stellar motions and lead to the relaxation of the system. In the course of relaxation, the stars, whose mass range spans 2-3 orders of magnitude, are driven toward equipartition. However, equipartition cannot be achieved in the presence of a central concentration of mass (in particular a central MBH). When two stars, which are initially on the same orbit (and therefore have the same velocity) interact, the massive one will slow down and the lighter one will speed up. Since the radius of the orbit depends only on the star's specific energy, and not its total energy, the massive star will sink to the center, while the lighter star will drift outwards. Over time, this process leads to "mass segregation" - the more massive stars are concentrated near the $\mathrm{MBH}$ and the lighter stars are pushed out of the inner region.

Occasionally, two-body interactions will eject a star out of the system altogether, thereby taking away positive energy from the system. The system will then become more bound and compact, the collision rate will increase, more stars will be ejected, and the result will be a runaway process. This process is called the "gravothermal catastrophe", or "core collapse", and is linked to the fact that self-gravitating systems have a negative heat capacity - they become hotter when energy is taken out. Core collapse, if unchecked, will lead to the formation of an extremely dense stellar core 
surrounded by a diffuse extended halo.

Once the density becomes high enough, very short range inelastic collisions are no longer extremely rare, and the fact that the stars are not point masses but have internal degrees of freedom starts to play a role. In such collisions energy is extracted from the orbit and invested in the work required to raise tides on the stars, or strip mass from them. The tidal energy is eventually dissipated in the star and radiated away. If the collision is slow, as it is in the core of a globular cluster where there is no $\mathrm{MBH}$, then the typical initial orbit is just barely unbound. In this case, the tidal interaction may extract enough orbital energy for "tidal capture", and lead to the formation of a tightly bound, or "hard" binary (tight, because tidal forces become effective only when the two stars are very close to each other). Hard binaries are a heat source for the cluster and play a crucial role in arresting core collapse. When a third star collides with a hard binary, it will tend to gain energy from the binary, thereby injecting positive energy to the cluster, while the binary becomes harder still.

When the stars orbit a central MBH, the collisions are fast (The Keplerian velocity near the MBH exceeds the escape velocity from the star) and the initial orbits are very unbound (hyperbolic). Even very close flybys cannot take enough energy from the orbit to bind the two stars, and so they continue on their way separately after having extracted energy and angular momentum from the orbit. The stars can radiate away the excess heat on a time scale shorter than the mean time between collisions, but it is harder to get rid of the excess angular momentum. Magnetic breaking (the torque applied to a star when the stellar wind resists being swept by the rotating stellar magnetic field), typically operates on time scales similar to the stellar lifetime. It is therefore likely that high rotation is the longest-lasting dynamical after effect of a close hyperbolic encounter, and that stars in a high density cusp are spun-up stochastically by repeated collisions (\$1.3.2). Finally, at zero range, almost head-on stellar collisions can lead to the stripping of stellar envelopes (\$1.3.1), the destruction of stars, or to mergers that result in the creation of "exotic stars". These are stars that cannot be formed in the course of normal stellar evolution, such as a Thorne-Zytkow object, which is an accreting neutron star embedded in a giant envelope (Thorne \& Zytkow 1975).

\subsubsection{Physical scales}

There are several important timescales and lengthscales that govern the dynamics of the stellar system and MBH. They are listed here with estimates of their value in the Galactic Center. A solar type star and $M_{\bullet}=3 \times 10^{6} M_{\odot}$ (Genzel et al. 2000) are assumed throughout. Physical lengths are expressed also as angular sizes assuming that the distance to the Galactic Center is $R_{0}=8 \mathrm{kpc}($ Reid 1993). 


\subsubsection{Timescales}

The dynamical time, or orbital time, $t_{d}$, is the time it takes a star to cross the system

$$
t_{d} \sim \frac{r}{v} \sim 2 \pi \sqrt{\frac{\mathrm{r}^{3}}{G M_{\mathrm{tot}}}} \sim 2 \times 10^{5} \mathrm{yr}(\text { at } 3 \mathrm{pc}) \sim 300 \mathrm{yr}(\text { at } 0.03 \mathrm{pc}),
$$

where $r$ is the typical size of the system and $M_{\text {tot }}$ is the total mass enclosed in radius $r$.

The 2-body relaxation time, $t_{r}$, is related to the $1 \mathrm{D}$ velocity dispersion $\sigma$, the mean stellar mass $\left\langle M_{\star}\right\rangle$ and the stellar number density $n_{\star}$ by

$$
t_{r} \sim \frac{0.34 \sigma^{3}}{G^{2}\left\langle M_{\star}\right\rangle^{2} n_{\star} \ln \Lambda} \sim 10^{9} \mathrm{yr},
$$

where $\log \Lambda$ is the Coulomb logarithm, the logarithm of the ratio between the largest and smallest impact parameters possible in the system for elastic collisions. Because the relaxation timescale in the Galactic Center is shorter than the age of the Galaxy ( $\sim 10 \mathrm{Gyr})$, the old stars are expected to be well relaxed by now.

The mass segregation timescale is of the same order as the relaxation timescale,

$$
t_{\mathrm{seg}} \sim t_{r}
$$

The rate (per star) of grazing collisions between two stars of mass and radius $M_{\star}^{a}, R_{\star}^{a}$ and $M_{\star}^{b}, R_{\star}^{a}$, each, is

$t_{c}^{-1}=4 \sqrt{\pi} n_{\star} \sigma\left(R_{\star}^{a}+R_{\star}^{b}\right)^{2}\left[1+\frac{G\left(M_{\star}^{a}+M_{\star}^{b}\right)}{2 \sigma^{2}\left(R_{\star}^{a}+R_{\star}^{b}\right)}\right] \sim 10^{-9} \mathrm{yr}^{-1}($ at $0.03 \mathrm{pc})$,

where it is assumed that the stars follow a mass independent MaxwellBoltzmann velocity distribution with velocity dispersion $\sigma$ (this is a good approximation near the $\mathrm{MBH}$, see $\$ 1.2 .2$ ). There are two contributions to the total rate, one due to the geometric cross-section (first term in the square brackets) and one due to "gravitational focusing" (second term in the square brackets). Gravitational focusing expresses the fact that the two stars do not move on straight lines, but are attracted to each other. This effect is important when the typical stellar velocities are much smaller than the escape velocity from the stars, $\sigma^{2}<G M_{\star} / 2 R_{\star}=v_{\text {esc }}^{2} / 4$.

\subsubsection{Lengthscales}

The size of the event horizon of a non-rotating black hole, the Schwarzschild radius, is

$$
r_{s}=\frac{2 G M_{\bullet}}{c^{2}}=9 \times 10^{11} \mathrm{~cm} \sim 3 \times 10^{-7} \mathrm{pc} \sim 8 \mu \operatorname{arcsec} .
$$


The tidal radius, $r_{t}$, is the minimal distance from the $\mathrm{MBH}$ where the stellar self-gravity can still resist the tidal forces of the MBH. If the star's orbit takes it inside the tidal radius, it will be disrupted, and roughly half of its mass will fall into the MBH, while the other half will be ejected (e.g. Ayal, Livio \& Piran 2001). The exact value of the tidal radius depends on the stellar structure and the nature of the orbit, and up to a factor of order unity is given by

$$
r_{t} \sim R_{\star}\left(\frac{M_{\bullet}}{M_{\star}}\right)^{1 / 3}=10^{13} \mathrm{~cm} \sim 3 \times 10^{-6} \mathrm{pc} \sim 80 \mu \operatorname{arcsec} .
$$

Tidal disruption is relevant as long as the tidal radius lies outside the event horizon. Since $r_{t} \propto M_{\bullet}^{1 / 3}$, while $r_{s} \propto M_{\bullet}$, there exists a maximal MBH mass for tidal disruption, which for a solar type stars is $\sim 10^{8} M_{\odot}$.

The radius of influence, $r_{h}$, is the region where the $\mathrm{MBH}$ potential dominates the dynamics. If the $\mathrm{MBH}$ is embedded in an isothermal stellar system (i.e. $\sigma$ is constant), then the radius of influence can be defined as

$$
r_{h}=\frac{G M_{\bullet}}{\sigma^{2}} \sim 10^{19} \mathrm{~cm} \sim 3 \mathrm{pc} \sim 80 \operatorname{arcsec} .
$$

In practice, the distribution is not isothermal and $\sigma$ is not constant, and so $r_{h}$ is evaluated loosely by choosing a representative value of $\sigma$ far enough

from the MBH. The stellar mass enclosed within $r_{h}$ is of the same order as the mass of the $\mathrm{MBH}$.

\subsubsection{A relaxed stellar system around a MBH}

The relaxed, quasi steady-state density distribution of a single-mass stellar population around a $\mathrm{MBH}$ is (Bahcall \& Wolf 1976; see also Binney \& Tremaine 1987 for a simple derivation)

$$
n_{\star} \propto r^{-7 / 4} .
$$

When the the stellar population consists of a spectrum of masses, $M_{1}<$ $M_{\star}<M_{2}$, the stellar distribution function (DF) very near the MBH has the form (Bahcall \& Woll1977)

$$
f_{M}(\epsilon) \propto \epsilon^{p_{M}}, \quad n_{\star} \propto r^{-3 / 2-p_{M}}, \quad p_{M} \equiv \frac{M}{4 M_{2}},
$$

where $-\epsilon$ is the total specific energy of the star and $f_{M} \equiv 0$ for $\epsilon<0$. The velocity dispersion of this DF (see equation 1.12 below) is almost independent of the stellar mass,

$$
\sigma_{M}^{2}=\left(\frac{1}{5 / 2+p_{M}}\right) \frac{G M_{\bullet}}{r},
$$




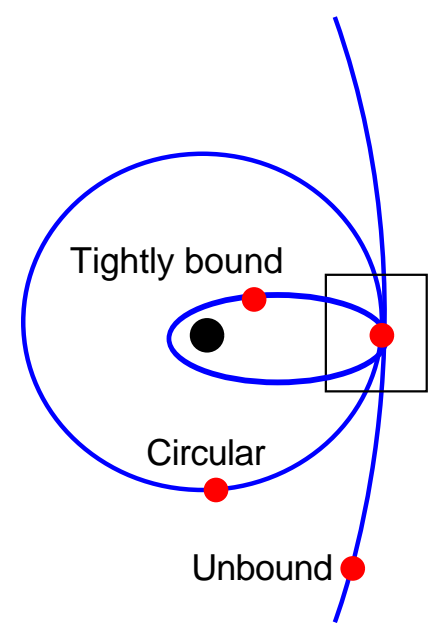

Figure 1.1. A schematic representation of the different types of stellar orbits that can be observed in a small region near the $\mathrm{MBH}$.

which implies that $\sigma_{M}^{2}$ changes by less than $10 \%$ over the entire mass range, in marked contrast to the $\sigma_{M}^{2} \propto M_{\star}^{-1}$ dependence of equipartition. This result justifies the approximation that the velocity dispersion in a relaxed stellar system around a MBH is mass-independent.

The Bahcall-Wolf solution applies to point particles. This assumption no longer holds very near the $\mathrm{MBH}$, where the collision rate is high because of the very high stellar density. Stars on tight orbits around the $\mathrm{MBH}$ cannot survive for long, and so eventually most of the population there will consist of stars that are on very wide, marginally bound (parabolic) orbits, which spend only a small fraction of their time in the collisionally dominated region. These marginally bound stars have a flatter spatial distribution, of the form (e.g. Binney \& Tremaine 1987, p. 551)

$$
n_{\star} \propto r^{-1 / 2} .
$$

The stars in any volume element near the MBH have a distribution of orbits (Figure 1.1): some are more bound than circular (i.e. their velocity is smaller than the circular velocity $v_{c}$ ), some are less bound than circular, some are unbound to the $\mathrm{MBH}$ (but bound by the total mass of the $\mathrm{MBH}$ and stars). The distribution of orbits is directly tied to the spatial distribution through the Jeans Equation,

$$
\frac{G M_{\bullet}}{r \sigma^{2}}=\frac{v_{c}^{2}}{\sigma^{2}}=-\frac{d \ln n_{\star}}{d \ln r}-\frac{d \ln \sigma^{2}}{d \ln r} .
$$




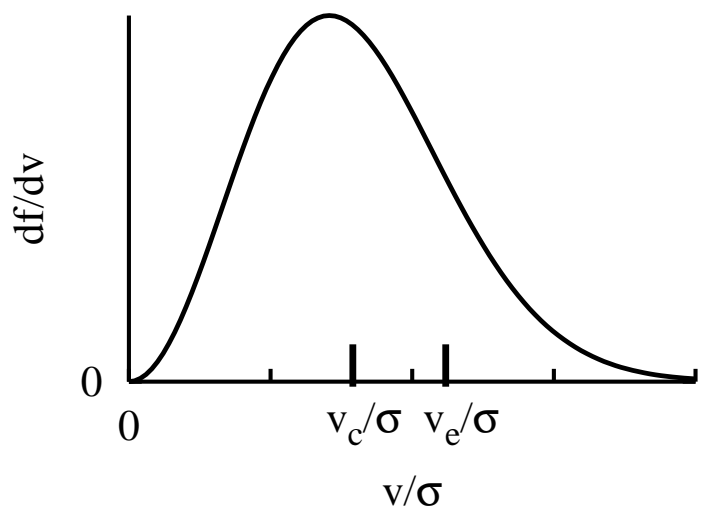

Figure 1.2. The fraction of orbits in the Maxwell-Boltzmann distribution as function of the normalized velocity $v / \sigma$. The circular velocity $v_{c}$ and the escape velocity $v_{e}=\sqrt{2} v_{c}$ are marked for a density distribution with $\alpha=1.5$. The region $v>v_{e}$ lies under the exponential tail of the $\mathrm{DF}$, and so the fraction of stars with unbound orbits is a strongly decreasing function of $\alpha$.

The Jeans equation is essentially a re-statement of the continuity equation of the stellar orbits in phase space in terms of averaged quantities, the mean stellar density and velocity dispersion. Here it is given for the simplest case of a steady state, isotropic, non-rotating system. The steady-state assumption is justified because the dynamical timescale is much shorter than the relaxation timescale. The assumptions of isotropy and non-rotation are observationally justified.

Very near the MBH the velocity dispersion is Keplerian, $\sigma^{2} \propto r^{-1}$, and so for any power-law cusp $n_{\star} \propto r^{-\alpha}$ the Jeans equation implies that

$$
\frac{v_{c}^{2}}{\sigma^{2}}=\alpha+1
$$

The steeper the cusp (larger $\alpha$ ), the larger the ratio between $v_{c}$ and $\sigma$, and so the fraction of loosely bound stars or unbound stars is smaller (Figure 1.2). Because unbound stars have wide orbits and spend most of their time far away from the $\mathrm{MBH}$, the stellar population in a shallow cusp is well mixed and representative of the average population over a large volume. In contrast, the stellar population in a steep cusp is localized and can therefore develop and maintain properties that differ from those of the general population. 


\subsection{The stellar collider in the Galactic Center}

The potential for probing a new regime of stellar dynamics near the $\mathrm{MBH}$ in the Galactic Center is best illustrated by comparing the collisional timescale there with that in the cores of the densest globular clusters, which for long served as laboratories for the study of collisional processes. In a dense globular cluster, $n_{\star} \sim 10^{6} M_{\odot} \mathrm{pc}^{-3}$ and $\sigma \sim 10 \mathrm{kms}^{-1}$, whereas in the Galactic Center, the density may be as high as $n_{\star} \sim 10^{8} M_{\odot} \mathrm{pc}^{-3}$ (\$1.3.1) and $\sigma \sim 1000 \mathrm{kms}^{-1}$. The timescale for collisions between solar type stars in a globular cluster can be estimated from equation (1.4) to be almost $10^{10} \mathrm{yr}$, roughly the age of the Galaxy and of a solar type star, whereas it is only $\sim 5 \times 10^{8} \mathrm{yr}$ in the inner $0.02 \mathrm{pc}$ of the Galactic Center. These estimates imply that while physical collisions are only marginally relevant in the cores of the densest globular clusters, they completely dominate the dynamics in the innermost part of the $\mathrm{MBH}$ cusp

\subsubsection{The case for a dense stellar cusp in the Galactic Center}

Theoretical expectations lead us to expect a relaxed stellar cusp around the $\mathrm{MBH}$ in the Galactic Center. Does such a cusp indeed exist there? The answer depends critically on the problem of identifying which of the observed stars are dynamically relaxed, since only those faithfully trace the underlying old stellar population. The analysis presented here shows that it is possible to interpret the available observations self-consistently in the framework of a high density cusp. However, the reader should keep in mind that the issue is an empirical one, and as such may be subject to revisions when more and better data is obtained about the stars near the $\mathrm{MBH}$.

Direct evidence for the existence of a cusp comes from the analysis of star maps, which show a concentration of stars toward the center. Assuming a 3D density distribution of the form $n_{\star} \propto r^{-\alpha}$, the corresponding projected $2 \mathrm{D}$ surface density can be compared to the observed distribution to find the most likely value of $\alpha$. Figure 1.3 shows the likelihood curves for $\alpha$ based on three independent star maps, after all the stars that were spectroscopically identified as young were taken out of the sample (the faint blue stars nearest to $\operatorname{Sgr} A^{\star}$ are included only in the Keck data set, but not in the other two). The most likely value for the density power-low index $\alpha$ lies in the range $\sim 1.5-1.75$. A flat core $(\alpha \sim 0)$, such as exists in globular clusters, is decisively rejected. Similarly, a likelihood test for the maximal size of a flat inner core indicates that such a core, if it exists, is be smaller than $\sim 0.1 \mathrm{pc}\left(2.5^{\prime \prime}\right)$. It can be shown that extinction by interstellar dust is unlikely to bias these results by a significant amount.

Additional evidence for the existence of a very high density cusp comes from the observed gradual depletion of the luminous giants toward the

1 The probability for avoiding a collision over a time $t$ is $\exp \left(-t / t_{c}\right)$. 


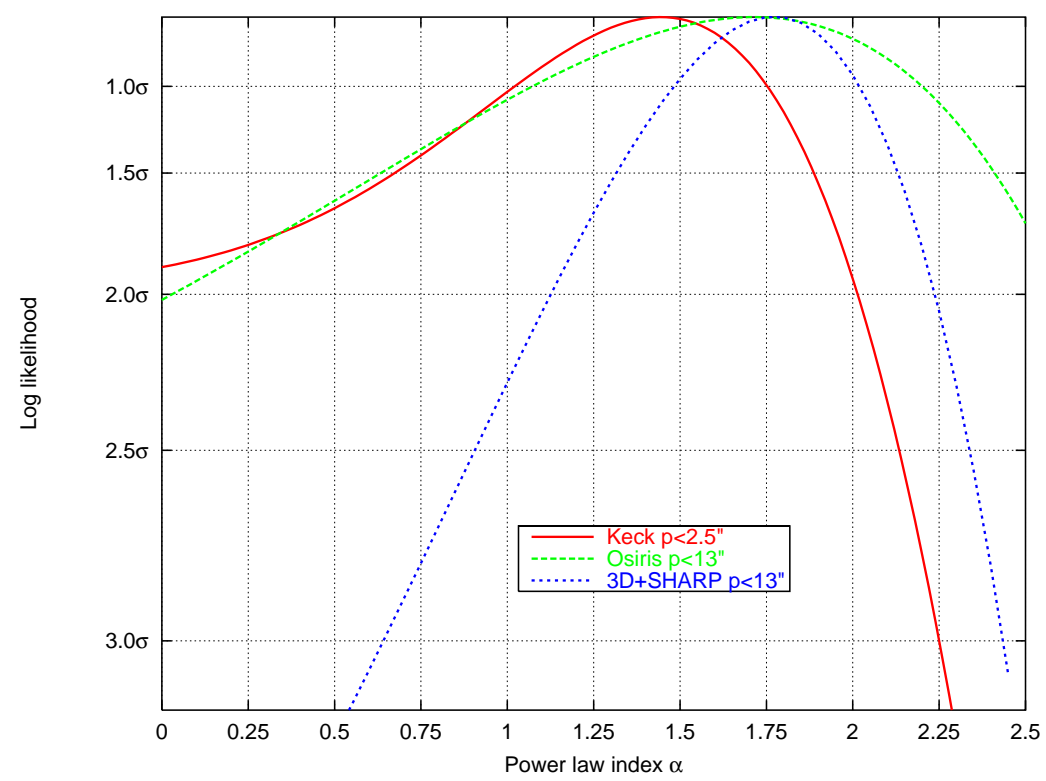

Figure 1.3. A Maximum likelihood analysis of the surface density distribution of stars near $\mathrm{SgrA}^{\star}$ for a 3D stellar density distribution $n_{\star} \propto r^{-\alpha}$ (Alexander 1999). Three different data sets (Blum et al. 1996; Genzel et al. 1996; Eckart \& Genzel 1997; Ghez et al. 1998) indicate that the most likely value for $\alpha$ lies in the range $\sim 3 / 2$ to $\sim 7 / 4$, which is the theoretically predicted range for a relaxed stellar system around a MBH (Bahcall \& Wolf 1977). Order of magnitude estimates $(\$ 1.2 .1)$ suggest that the stellar system around the $\mathrm{MBH}$ in the Galactic Center has undergone two-body relaxation. (Reprinted with permission from The Astrophysical Journal).

MBH in the inner $0.1 \mathrm{pc}$ (Figure 1.4). Luminous red giants have very large extended envelopes, and therefore a large cross-section for collisions with other stars. When the impact parameter is a small fraction of the giant's radius, the envelope may be stripped, leaving behind an almost bare burning core. This will make the star effectively invisible in the infrared (IR) because the IR spectral range lies in the Raleigh-Jeans part of the stellar blackbody spectrum, and so the IR luminosity scales as $L_{\mathrm{IR}} \propto R_{\star}^{2} T_{\text {eff }}$ while the total luminosity scales as $L_{\star} \propto R_{\star}^{2} T_{\text {eff }}^{4}$. Suppose that the collision disperses the envelope of a $\sim 100 R_{\odot}$ red supergiant and leaves a $\sim 1 R_{\odot}$ burning core. In order to maintain the total stellar luminosity, the effective temperature will have to rise by a factor 10 , which will result in a decrease of the IR luminosity by a factor of 1000 (7.5 magnitudes). 


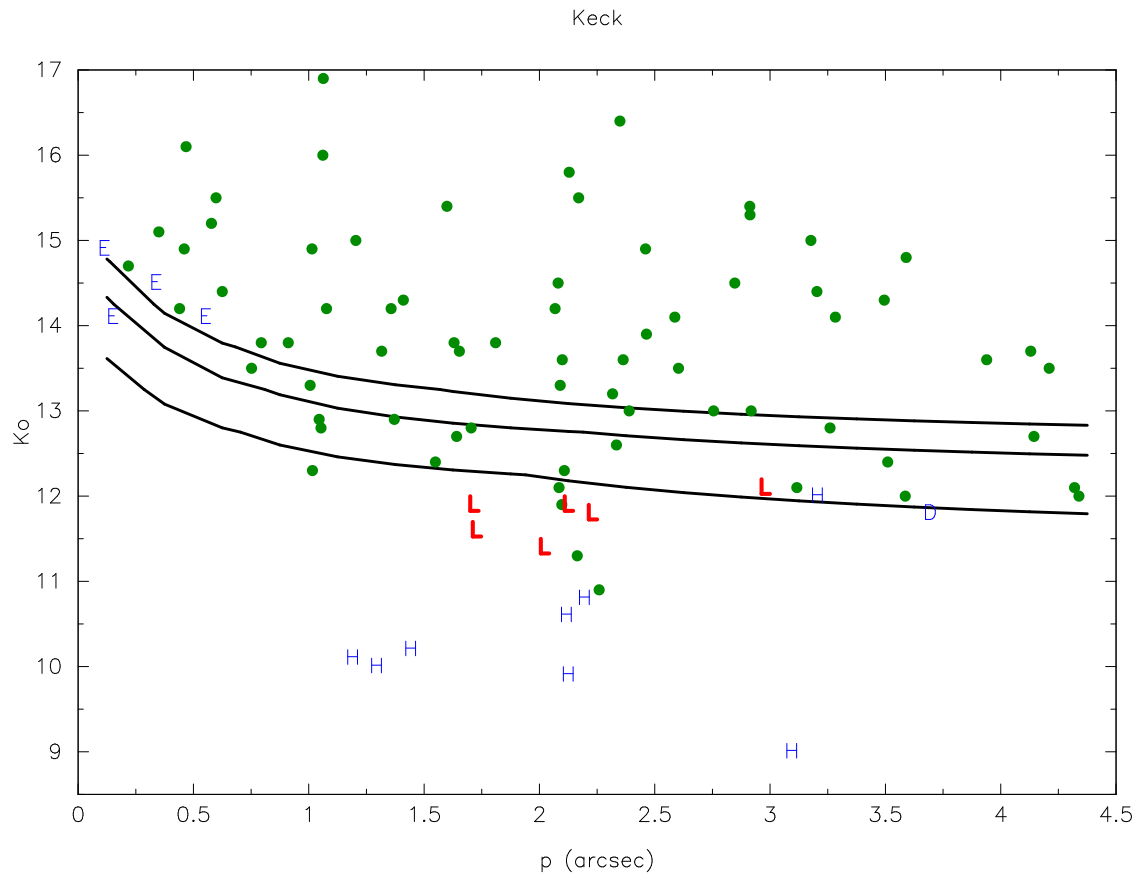

Figure 1.4. Evidence for collisional destruction of bright giant envelopes in a high density stellar cusp around the $\mathrm{MBH}$ in the Galactic Center (Alexander 1999). The apparent stellar $K$-band magnitude is plotted against the projected angular distance from the black hole, $p$ (Keck data from Ghez et al. 1998). The ages of the stars marked by circles are unknown, but it is likely that most of them are old, and therefore dynamically relaxed. Stars marked by "L" are spectroscopically identified as old stars. Stars marked by "H" are spectroscopically identified as young stars and are not dynamically relaxed. Such stars are not expected to be affected by collisions because of their short lifetimes. The stars marked by "E" have featureless blue spectra and are either young stars or old stars that were affected by the extreme conditions very near the black hole. The three contour lines represent detailed model predictions for the decrease in surface density of bright stars due to collisional destruction in a high density $n_{\star} \propto r^{-3 / 2}$ stellar cusp. The stellar density reaches a value of $\sim 4 \times 10^{8} \mathrm{M}_{\odot} \mathrm{pc}^{-3}$ at $r=0.25^{\prime \prime}(0.01$ pc), which is 9 orders of magnitude higher than in the Solar Neighborhood, and almost 3 orders of magnitude higher than in the densest globular cluster core. The model predicts, on average, 1.5 (top contour), 1.0 (central contour), and 0.5 (bottom contour) dynamically relaxed stars per 0.25 arcsecond bin that are brighter than the contour level. This is consistent with the observed trend in the surface density distribution. (Reprinted with permission from The Astrophysical Journal). 
Figure 1.4 compares a theoretical prediction for the collisional depletion of luminous giants with the data. The match with the observed trend is remarkably good, given the fact that no attempt was made to fit the data. The calculation is based on detailed modeling of expected numbers, sizes, luminosities and lifetimes of giants in the population, on cross-sections for envelope disruption that were calibrated by hydrodynamical simulations, and on a stellar density cusp that is normalized by dynamical estimates of the enclosed mass.

It should be noted that the total mass loss rate from these collisions is smaller than that supplied by the strong stellar winds of the blue supergiants in the inner few arcseconds, and so stellar collisions are not a dominant source of mass supply to the MBH at this time.

The self-consistent picture that emerges from this analysis is that the stars near the $\mathrm{MBH}$ in the Galactic Center, which are expected to be dynamically relaxed, are indeed concentrated in a stellar cusp of the form predicted by theory for a relaxed system. The very high stellar density in the inner few $0.01 \mathrm{pc}$ leads to frequent collisions that destroy the envelopes of giant stars, thereby explaining the gradual depletion in the number of luminous giants toward the center. The central cluster of faint blue stars in the inner $0.5^{\prime \prime}$ coincides with the collisionally dominated region. It is therefore relevant to consider dynamical explanations for their nature and appearance as an alternative to assuming that they are newly formed, unrelaxed stars. The concentration of such a distinct population in a small volume is consistent with the tightly bound orbits that are typical of a steep cusp (\$1.2.2).

\subsubsection{Tidal spin-up}

It is inevitable that in a system where the stellar density is high enough for collisional destruction of giants, smaller stars that escape destruction will still suffer very close encounters. As described above ( $\$ 1.2)$, usually such collisions cannot bind the two stars, and the longest lasting after effect, apart from possible mass loss, is fast rotation. Fast rotation and mass loss have the potential to affect stellar evolution and modify the appearance of the stars (see discussion in Alexander \& Kumar 2001; Alexander \& Livio 2001). Although detailed predictions of the observational consequences are still not available, it is of interest to estimate the magnitude of the spin-up effect.

When the tidal deformations in the star are small, the change in the angular velocity of a star of mass $M_{\star}$ and radius $R_{\star}$ due to an encounter with a mass $M$ can be described by a linear multipole expansion in the periapse distance $r_{p}$ (distance of closest approach) by (Press \& Teukolsky 1977) 


$$
\Delta \widetilde{\Omega}=\frac{\widetilde{M}^{2}}{\widetilde{I} \widetilde{v}_{p}} \sum_{l=2}^{\infty} \frac{T_{l}(\eta, e)}{\widetilde{r}_{p}^{l+1}},
$$

where the tilde symbol denotes quantities measured in units of $G=M_{\star}=$ $R_{\star}=1$, and rigid body rotation is assumed. $\widetilde{v}_{p}$ is the relative velocity at periapse, $\widetilde{I}$ is the star's moment of inertia, and $T_{l}$ the tidal coupling coefficient of the $l$ 'th moment. In these units, $\widetilde{\Omega}=1$ is the centrifugal breakup angular velocity, where the star sheds mass from its equator. The tidal coupling coefficients depend on the star's structure and on the orbital parameters through the quantity $\eta=\widetilde{r}_{p}^{3 / 2} / \sqrt{1+\widetilde{M}}$ and the orbital eccentricity $e$. The tidal coefficient $T_{l}$ can be calculated numerically for any given stellar model and orbit.

The formal divergence of $\Delta \widetilde{\Omega}$ as $\widetilde{r}_{p}$ decreases indicates that most of the contribution comes from close collisions, where the linear analysis breaks down. The non-linear processes, which truncate the divergence, have to be investigated by hydrodynamical simulations (see $\$ 1.3 .3)$. These reveal that as $\widetilde{r}_{p}$ decreases towards $1, \Delta \widetilde{\Omega}$ first increases faster than predicted by the linear analysis, but then it reaches a maximal value at the onset of mass loss, since the ejecta carry away the extra angular momentum.

Over its lifetime, a star will undergo many tidal encounters, randomly orientated relative to its spin axis, and will be spun-up in a random walk manner. The cumulative effect can be large. Figure 1.5 shows the predicted average spin-up of a solar type stars over 10 Gyr in the Galactic Center as function of distance from the MBH (Alexander \& Kumar 2001). The calculation assumes an $\alpha=1.5$ density cusp, a model for the distribution of stellar masses in the population, and inefficient magnetic breaking. On average, solar type stars in a large volume around the black hole are spunup to $10 \%-30 \%$ of the break-up angular velocity, or 20 to 60 times faster than is typical in the field. The effect falls off only slowly with distance because the higher efficiency of tidal interactions in slower collisions far from the black hole offsets the lower collision rate there.

\subsubsection{Tidal scattering}

Tidal scattering is another mechanism that can affect the internal structure of a significant fraction of the stars around the $\mathrm{MBH}$. Unlike the tidal spinup process discussed in $\S 1.3 .2$, tidal scattering does not require a very high stellar density, since it is driven by the global response of the system to the existence of a mass sink, the MBH, in its center.

Some of the mass that feeds the growth of a MBH in a galactic center is supplied by tidal disruption of stars that are scattered into low angular momentum orbits ("loss-cone" orbits). When the MBH mass is small enough so that the tidal radius is larger than the event horizon, $r_{t}>r_{\mathrm{s}}$, 


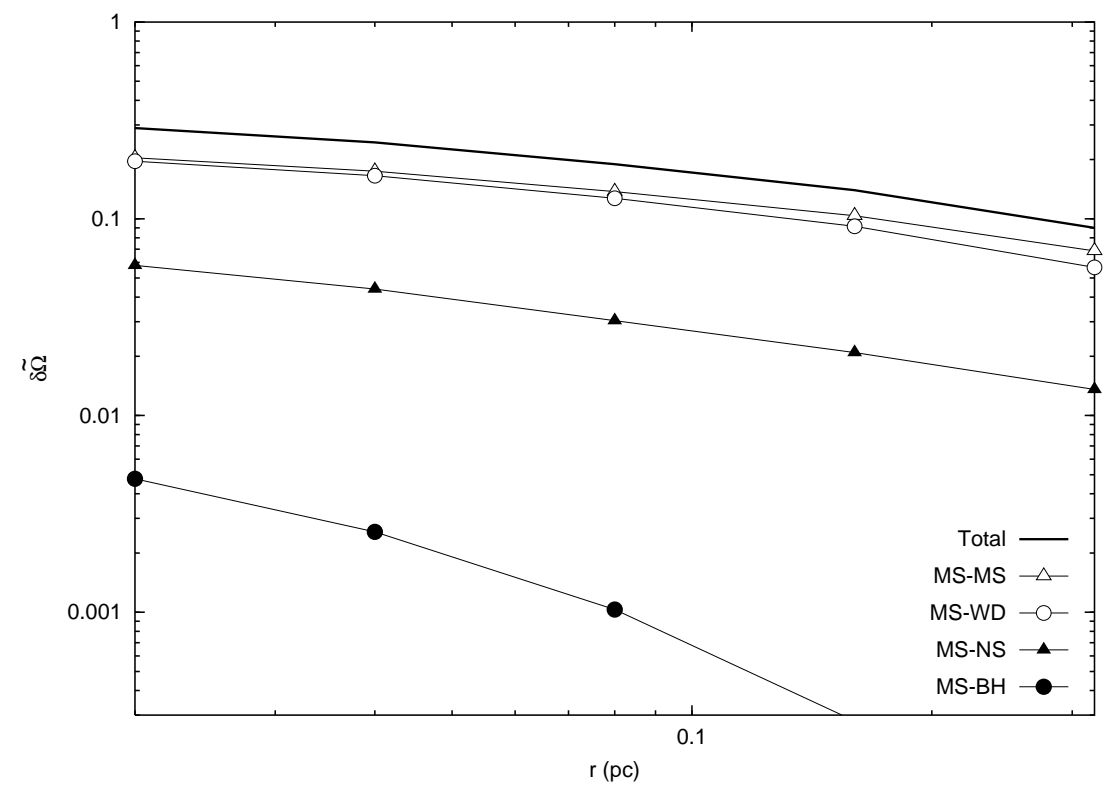

Figure 1.5. The average spin-up of a solar type star by star-star tidal interactions over $10 \mathrm{Gyr}$ as function of distance from the black hole in the Galactic Center (Alexander \& Kumar 2001). An $\alpha=1.5$ density cusp is assumed. The rotation grows over time in a random walk fashion by repeated close passages. $\delta \widetilde{\Omega}=1$ corresponds to rotation at the centrifugal break-up velocity. In addition to the total spin-up, the separate contributions from collisions with main sequence stars (MS), white dwarfs (WD), neutron stars (NS) and stellar black holes (BH) are shown. (Reprinted with permission from The Astrophysical Journal).

the star is tidally disrupted before crossing the event horizon. The accretion of stellar debris from such events may give rise to observable "tidal flares" (Frank \& Rees 1976). Significant theoretical efforts have gone into estimating the rates, timescales, luminosities and spectra of the flares (e.g. Ulmer, Paczyński, \& Goodman, 1998; Magorrian \& Tremaine 1999; Ayal, Livio \& Piran 2001), in the hope that they can be used to detect MBHs in the centers of galaxies. There is today only marginal evidence for the detection of such flares (e.g. Renzini et al. 1995; Komossa \& Bade 1999; Komossa \& Greiner 1999).

The effect of MBH's tidal field is not limited to tidal disruption. For every star that is actually disrupted, there are stars with $r_{p} \gtrsim r_{t}$ that narrowly escape tidal disruption by the central $\mathrm{BH}$ after being subjected to extreme tidal distortion, spin-up, mixing and mass-loss, which may affect their evolution and appearance (Alexander \& Livio 2001). Figure 1.6 shows 

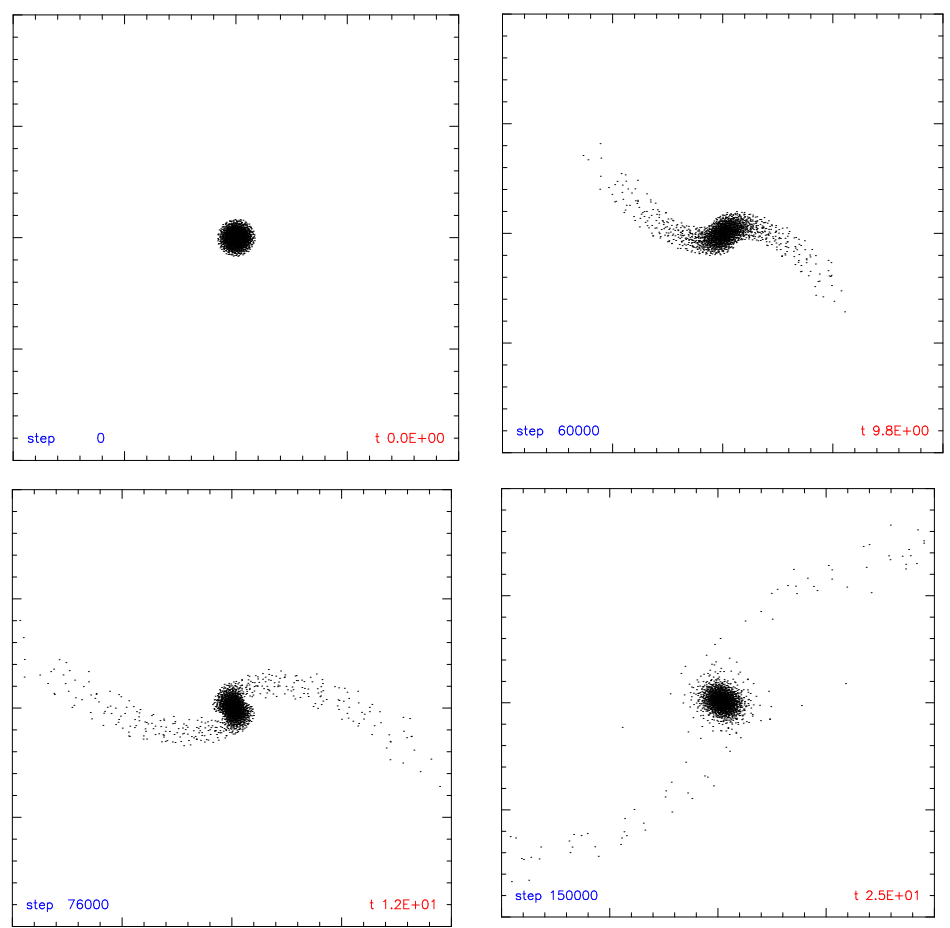

Figure 1.6. Snapshots from a Smoothed Particle Hydrodynamics (SPH) simulation of a star undergoing an extreme non-disruptive tidal interaction ("tidal scattering") as it passes near a massive black hole. Time is measured in units of the star's dynamical timescale. The star passes near the black hole (located outside of the frame) on a parabolic orbit with a peri-distance 1.5 times larger than the tidal disruption distance. Shortly after periapse passage $(t=12)$ the star appears to be on the verge of breaking in two. However, by the end of the simulation, the two fragments coalesce, leaving a distorted, mixed and rapidly rotating bound object.

\section{Smoothed Particle Hydrodynamics (SPH) simulation of a star passing}

$2 \mathrm{SPH}$ is an algorithm for simulating the hydrodynamics of 3D self-gravitating fluids, which is commonly used in the study of stellar collisions (Monaghan 1992). The star is represented by discrete mass elements, each distributed smoothly over a small sphere so that the density peaks in the center and falls to zero at the edge. The total density at a point is the sum of densities in all overlapping spheres that include the point. The resulting density field is continuous and differentiable, and so its thermodynamic properties can be evaluated everywhere once an equation of state is specified. Every time step, the positions of the mass elements are updated according to the gravitational force and the pressure gradient, and the sphere sizes are readjusted to reflect the changes in the local density. 
by an $\mathrm{MBH}$ just outside the tidal disruption radius. To leading order, the effects of tidal scattering are a function of the penetration parameter $\beta=r_{t} / r_{p}$ only, and are independent of the $\mathrm{MBH}$ mass,

$$
\Delta \widetilde{\Omega} \simeq \frac{T_{2}\left(\beta^{-3 / 2}\right)}{\sqrt{2} \widetilde{I}} \beta^{9 / 2},
$$

which follows from equation (1.14) for a parabolic orbit and for $M_{\bullet} \gg M_{\star}$. As will be argued below, a large fraction of these "tidally scattered" stars survive eventual orbital decay and disruption, and so remain in the system as relics of the epoch of tidal processes even after the $\mathrm{MBH}$ becomes too massive for tidal disruption.

Dynamical analyses of the scattering of stars into the loss-cone orbits (Lightman \& Shapiro 1977; Magorrian \& Tremaine 1999) show that tidally disrupted stars in galactic nuclei are typically on slightly unbound orbits relative to the $\mathrm{MBH}$ and that they are predominantly scattered into the loss-cone from orbits at the radius of influence of the $\mathrm{BH}, r_{h}$. The scattering operates on a timescale that is shorter than the dynamical timescale, and so the stars are scattered in and out of the loss-cone several times during one orbital period. Because of gravitational focusing, the cross-section for scattering into a hyperbolic orbit with periapse $\leq r_{p}$ scales as $r_{p}$, and not as $r_{p}^{2}$ (Hills 1975; Frank 1978), and so the number of stars with $r_{t} \leq r_{p} \leq 2 r_{t}$ equals the number of stars that were disrupted by the MBH.

Tidal disruption is an important source of mass for a low-mass $\mathrm{MBH}$ that accretes from a low-density galactic nuclear core, where mass loss from stellar collisions is small (e.g. Murphy, Cohn \& Durisen 1991). For the $\mathrm{MBH}$ in the Galactic Center, the total mass in disrupted stars can be $0.25 M_{\bullet}$ or even higher (Freitag \& Benz 2001, in preparation). Since the enclosed stellar mass within $r_{h}$ is also $\sim M_{\bullet}$, the tidally scattered stars comprise a significantly high fraction of the stellar population within the radius of influence of the $\mathrm{MBH}$.

After the first periapse passage, the tidally scattered star will be on a very eccentric orbit with a maximal radius (apoapse) of $\lesssim 2 r_{h}$. Since the two body interactions that scattered it into the eccentric orbit operate on a timescale that is shorter than the orbital period, there is a significant chance that the star will be scattered again off the orbit and miss the MBH. The chance of this happening is further increased by the Brownian motion of the $\mathrm{MBH}$ relative to the dynamical center of the stellar system. The amplitude of the Brownian motion is much larger than the tidal radius, and it proceeds on the dynamical timescale of the core (Bahcall \& Wolf 1976), which is comparable to the orbital period of the tidally disturbed stars. The orbits of the tidally scattered stars take them outside of $r_{h}$, where they are no longer affected by the relative shift between the $\mathrm{BH}$ and the stellar mass. Therefore, on re-entry into the volume of influence, their orbit 


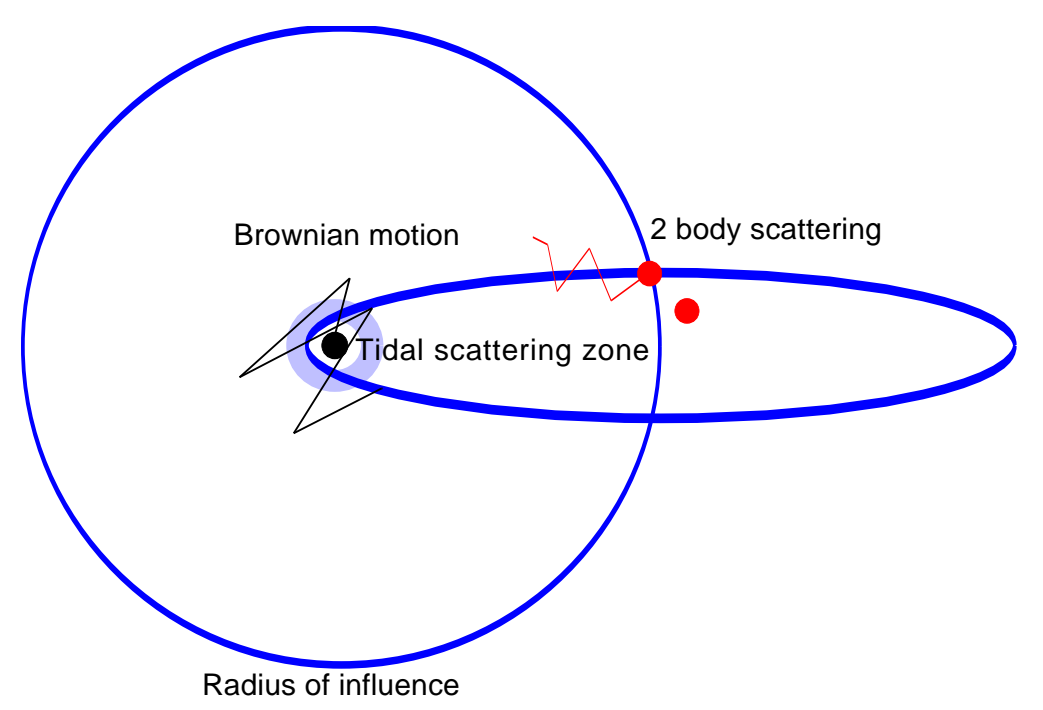

Figure 1.7. A schematic representation of the tidal scattering process. A star initially orbiting the $\mathrm{MBH}$ at the radius of influence is scattered by a two body encounter into an extremely eccentric orbit that brings it to the tidal scattering zone just outside the tidal disruption radius. The star suffers an extreme, non-disruptive tidal interaction with the $\mathrm{MBH}$, and continues on its way out of the radius of influence, where it is scattered by frequent two-body encounters. In the meanwhile, the Brownian motion of the $\mathrm{MBH}$ due to its interactions with the stellar system causes it to move away from its original position. Both these random processes significantly increase the chances of the tidally disturbed star to survive total disruption during subsequent orbits.

will not bring them to the same periapse distance from the $\mathrm{MBH}$. Both the random motion of the $\mathrm{MBH}$ and the scattering off the loss-cone by two-body interactions are expected to increase the survival fraction to a significantly high value. More detailed calculations, which integrate over the orbital distribution, are required to confirm these qualitative arguments.

Rough estimates (Alexander \& Livio 2001) indicate that the Galactic Center may harbor $10^{4-5}$ tidally scattered stars. These stars are expected to be on highly eccentric orbits, and so there may be observable correlations between high orbital eccentricity and the stellar properties.

\subsection{The gravitational telescope in the Galactic Center}

The MBH in the Galactic Center is a telescope with a lens of effective diameter $\sim 4 \times 10^{17} \mathrm{~cm}$ (for a source at infinity) and a focal length of 
$\sim 2.5 \times 10^{22} \mathrm{~cm}$. Unfortunately, Nature did not design it as an ideal telescope. A point mass lens does not produce faithful images of the lensed sources, the optical axis is heavily obscured by interstellar dust, and the telescope points in a fixed direction, which is not of our choosing. In fact, various estimates suggest that there are not enough luminous sources in that direction for gravitational lensing to be important for present day observations, although future, deep observations may pick up lensing events (Wardle \& Yusef-Zadeh 1992; Alexander \& Sternberg 1999; Alexander \& Loeb 2001). Nevertheless, it is worthwhile to consider the possible roles of gravitational lensing in the observations and study of the Galactic Center. This is important not only in anticipation of future observations, but also because the estimates of the lensing probability are quite uncertain (they involve models of the unobserved far side of the Galaxy), and because there are hints that lensing may not be quite as rare as predicted (\$1.4.2).

Gravitational lensing may be used to probe the dark mass (is it really a MBH?) and the stars around it, and to locate the MBH on the IR grid, where the stars are observed. On the other hand, gravitational lensing can also complicate the interpretation of the observations since it affects many of the observed properties of the sources: flux, variability, apparent motion and surface density. IR flares due to lensing can be confused with those due to fluctuations in the accretion flow, and lensed images of background sources far behind the MBH can be confused with stars that are truly near the MBH. This section will focus on aspects of gravitational lensing that are, or may be relevant for the Galactic Center. The reader is referred to Schneider, Ehlers \& Falco (1992) for a comprehensive treatment of the subject.

\subsubsection{Gravitational lensing by a point mass}

To first and good approximation the lensing properties of the mass distribution in the Galactic Center can be described as those of a point mass, the $\mathrm{MBH}$. Figure 1.8 shows the light ray diagram of lensing by a point mass in the small angle limit. The bending angle is given by

$$
\alpha=\frac{4 G M}{c^{2} b},
$$

where $b$ is the impact parameter of the light ray with respect to the lens. Note that unlike a glass lens, where the bending angle is zero when the ray goes through the lens center and increases with the impact parameter, the bending angle of a gravitational lens diverges towards the center and decreases with the impact parameter. It is therefore not surprising that a gravitational lens does not produce a faithful image of the lensed source, but rather breaks, warps and/or flips the image. A point lens creates two images of the source, one on either side of the lens. There are always two 


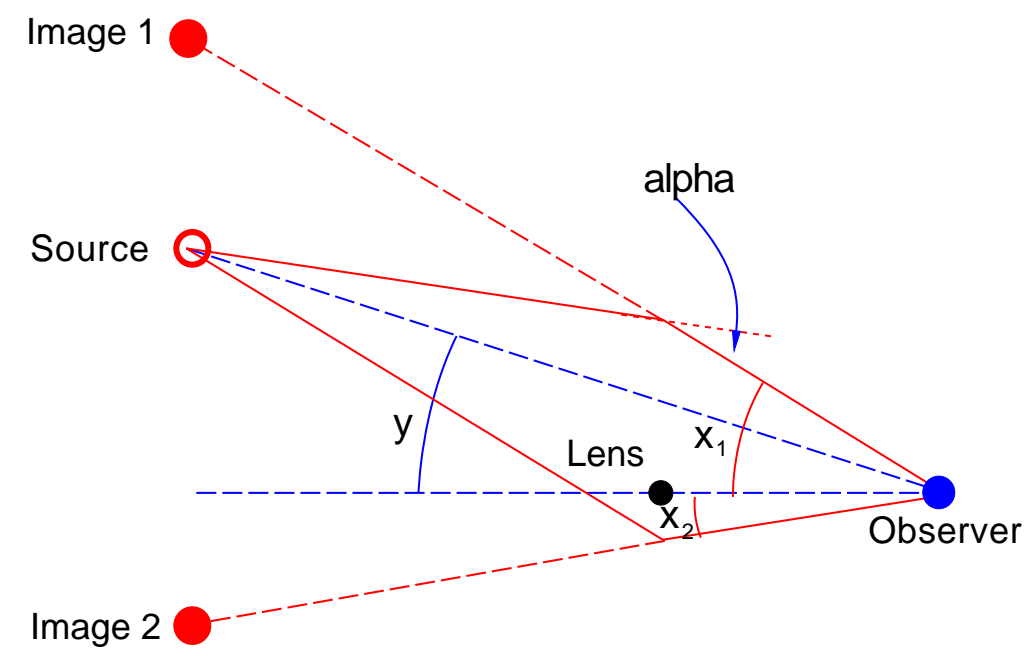

Figure 1.8. The light ray diagram for lensing by a point mass. The light rays from a source, at angular position $y$ relative to the observer-lens optical axis, are bent by the gravitational lens and reach the observer from angles $x_{1}$ and $x_{2}$, thereby appearing as two images. Unlike a glass lens, the light bending angle $\alpha$ of a gravitational lens is inversely proportional to the impact parameter to the lens (Equation 1.16).

images in focus at the observer, regardless of the distance of the source behind the lens. The two images, the lens and the (unobserved) source all lie on one line. The typical angular cross-section of the lens is given by the Einstein angle,

$$
\theta_{E}^{2}=\frac{4 G M_{\bullet}}{c^{2}} \frac{D_{L S}}{D_{O S} D_{O L}},
$$

where $D_{O L}$ is the observer-lens distance, $D_{L S}$ is the lens-source distance, and $D_{O S}$ is the observer-source distance 3 .

The relation between the angular position of the source relative to the observer-lens axis (the optical axis) can be derived from the geometry of the light paths,

$$
y=x_{1,2}-1 / x_{1,2},
$$

3 In flat spacetime, which is relevant for Galactic lensing, $D_{O S}=D_{O L}+D_{L S}$. In curved spacetime, which is relevant for cosmological lensing, the distances are the angular diameter distances, and this simple sum no longer holds. 
where $x_{1,2}$ and $y$ are measured in terms of $\theta_{E}$ and $x_{2}<0$ by definition. Gravitational lensing conserves surface brightness, and so the magnifications $A_{1,2}$ in the flux of each image relative to that of the unlensed source is proportional to change in the angular area of the source,

$$
A_{1,2}=\left|\frac{\partial \vec{y}}{\partial \vec{x}_{1,2}}\right|^{-1}=\left|1-x_{1,2}^{-4}\right|^{-1} .
$$

The primary image at $x_{1}$ is always magnified. The secondary image at $x_{2}$ can be demagnified to zero. The two magnifications obey the relations

$$
A_{1}=A_{2}+1 \geq 1
$$

and

$$
A \equiv A_{1}+A_{2}=\frac{y^{2}+2}{y \sqrt{y^{2}+4}} .
$$

When $y=0$ the amplification formally diverges and the image appears as a ring of angular size $\theta_{E}$, the Einstein ring. This divergence is avoided in practice by the finite size of the source (e.g. a star). Finite sized sources are also sheared tangentially around the Einstein ring as the magnification increases. In the limit of high magnification, or small source angle,

$$
A \sim 1 / y \quad(y \ll 1)
$$

\subsubsection{Pinpointing the $\mathrm{MBH}$ with lensed images}

Determining the exact position of the $\mathrm{MBH}$ on the IR grid is important because the radio source $\mathrm{SgrA}^{\star}$, which is associated with the $\mathrm{MBH}$, was detected to date only in one other band, the X-ray (Baganoff et al. 2001). Currently, the IR position of the radio source $\mathrm{SgrA}^{\star}$ is derived indirectly by aligning the radio and IR maps using 4 maser giants in the inner $15^{\prime \prime}$, which are observed in both bands (Menten et al. 1997). The exact IR position of the $\mathrm{MBH}$ is required, for example, for measuring the IR flux from the $\mathrm{MBH}$, in order to constrain accretion models; for solving the stellar orbits around the $\mathrm{MBH}$, in order to measure $M_{\bullet}$ and $R_{0}$ (Jaroszyński 1999; Salim \& Gould 1999) and search for general relativistic effects (Jaroszyński 1998; Fragile \& Mathews 2000; Rubilar \& Eckart 2001); and for detecting the fluctuations of the $\mathrm{MBH}$ away from the dynamical center of the stellar cluster, in order to study the stellar potential. Recent measurements of the acceleration vectors of three stars very near $\operatorname{Sgr} A^{\star}$ provide another way of locating the MBH (Ghez et al., 2000). The IR/radio alignment and the center of acceleration are close, but do not overlap (Figure 1.10), and neither coincide with an IR source. Gravitational lensing can provide a third, independent method for locating the $\mathrm{MBH}$. 


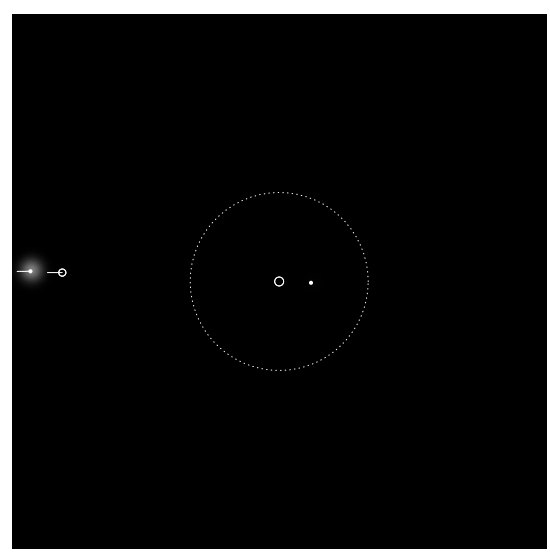

(a) $\mathrm{t}=-116$

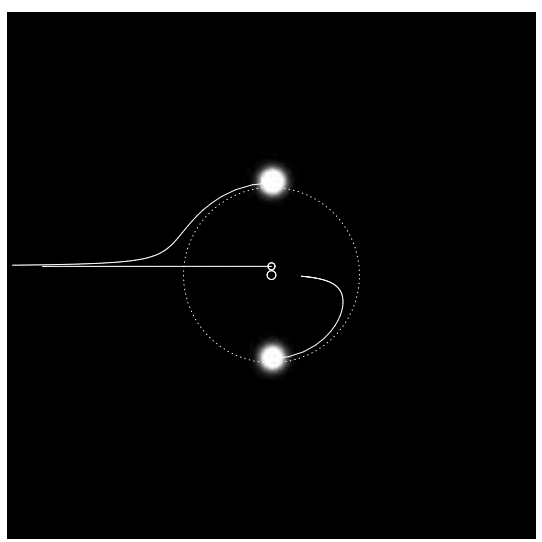

(c) $\mathrm{t}=0$

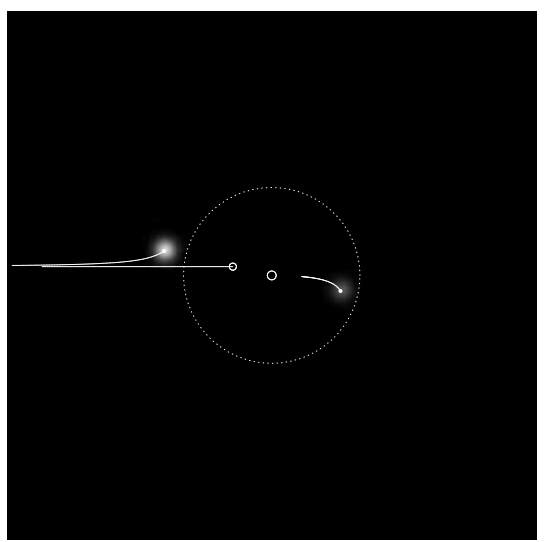

(b) $t=-11$

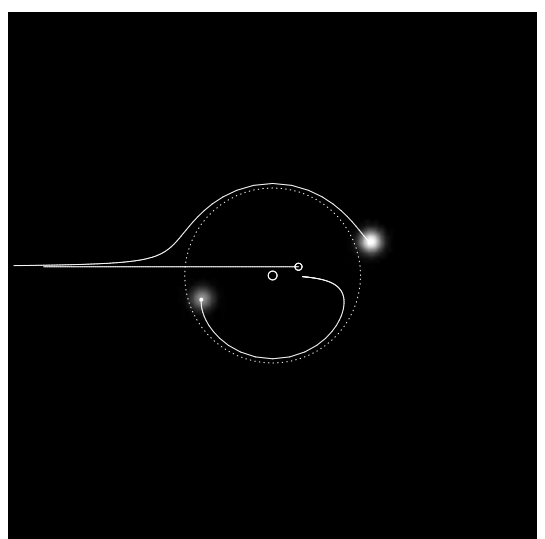

(d) $\mathrm{t}=+14$

Figure 1.9. A sequence of snapshots simulating the observation of lensing of a background point source by the MBH. Time is in arbitrary units. The background source, which is not observed directly, (open circle with straight line tracking the source trajectory) moves in projection from left to right behind the $\mathrm{MBH}$ (open circle at center) with an impact parameter of $0.1 \theta_{E}$. The two images (light points with curved lines tracking the image trajectories) move in tandem clockwise about the Einstein ring (large dotted circle). The strongly amplified image (top) is always outside the Einstein ring and is always brighter than the source. The weakly amplified image (bottom) is always inside the Einstein ring and can be strongly deamplified (panels a, b). At peak amplification (panel c) the two images are of comparable brightness (equation 1.20). 
When the source, lens and observer move relative to each other, the positions, velocities and magnifications of the images will change with time (Figure 1.9). In addition to the requirement that the two images and the lens lie on one line, equations (1.18) and (1.19) imply that the measured angular positions of the two images $\theta_{1,2}$, their projected transverse velocities $v_{t 1,2}$ and radial velocities $v_{r 1,2}$ relative to the lens, and their measured fluxes $F_{1,2}$, should obey the simple relation

$$
-\theta_{1} / \theta_{2}=v_{t 1} / v_{t 2}=-v_{r 1} / v_{r 2}=\sqrt{F_{1} / F_{2}} \text {. }
$$

The constraints are based solely on observables, and so are independent of any assumptions about $M_{\bullet}, R_{0}$ or the properties of the lensed background sources. The use of equation (1.23) does require knowledge of the exact position of the $\mathrm{MBH}$ relative to the stars, since this is needed for measuring the angular distances and for decomposing the radial and tangential components of the velocity. If the $\mathrm{MBH}$ position is known, equation (1.23) can be used to search in astrometric measurements of positions, fluxes and velocities for pairs of lensed images around the MBH. Equation (1.23) can also be used to find the position of the $\mathrm{MBH}$ on the IR grid, since the $\mathrm{MBH}$ lies on the line connecting the two images, and so the intersection of these lines pinpoints its position. This can be done statistically, by enumerating over a grid of trial positions for the $\mathrm{MBH}$, and choosing as the most likely one that which maximizes the number of lensed image pairs.

Figure (1.10) shows the results from such a joint statistical search for the $\mathrm{MBH}$ and for a signature of lensing (Alexander 2001). The most likely position of the $\mathrm{MBH}$ coincides with the center of acceleration. The random probability for such a likelihood extremum is 0.01 . The random probability for such an extremum to fall in either the $1 \sigma$ error range of $\mathrm{IR} /$ radio alignment or that of the center of acceleration is $5 \times 10^{-4}$.

The search for the $\mathrm{MBH}$ yields also a list of candidate lensed image pairs. The definitive test of lensing is to compare their spectra, which should be identical up to differences due to non-uniform extinction. Unfortunately, spectra for the fainter secondary images are unavailable at this time. Once $M_{\bullet}, R_{0}$ and the dust distribution in the Galaxy are assumed, it is possible to derive, albeit with very large uncertainty, the luminosity and distance of the candidate sources. The sources of the two most likely lensed image pair candidates are luminous supergiants, a blue supergiant a few kpc behind the Galactic Center and a red supergiant at the far edge of the Galaxy.

This statistical result, while intriguing, requires additional confirmation. Simple models of the distribution of light and dust in the Galaxy predict that the chances of finding luminous supergiants right behind the $\mathrm{MBH}$ are very small, and the statistical analysis depends sensitively on the quality of the data and its error properties. Whether or not this particular 


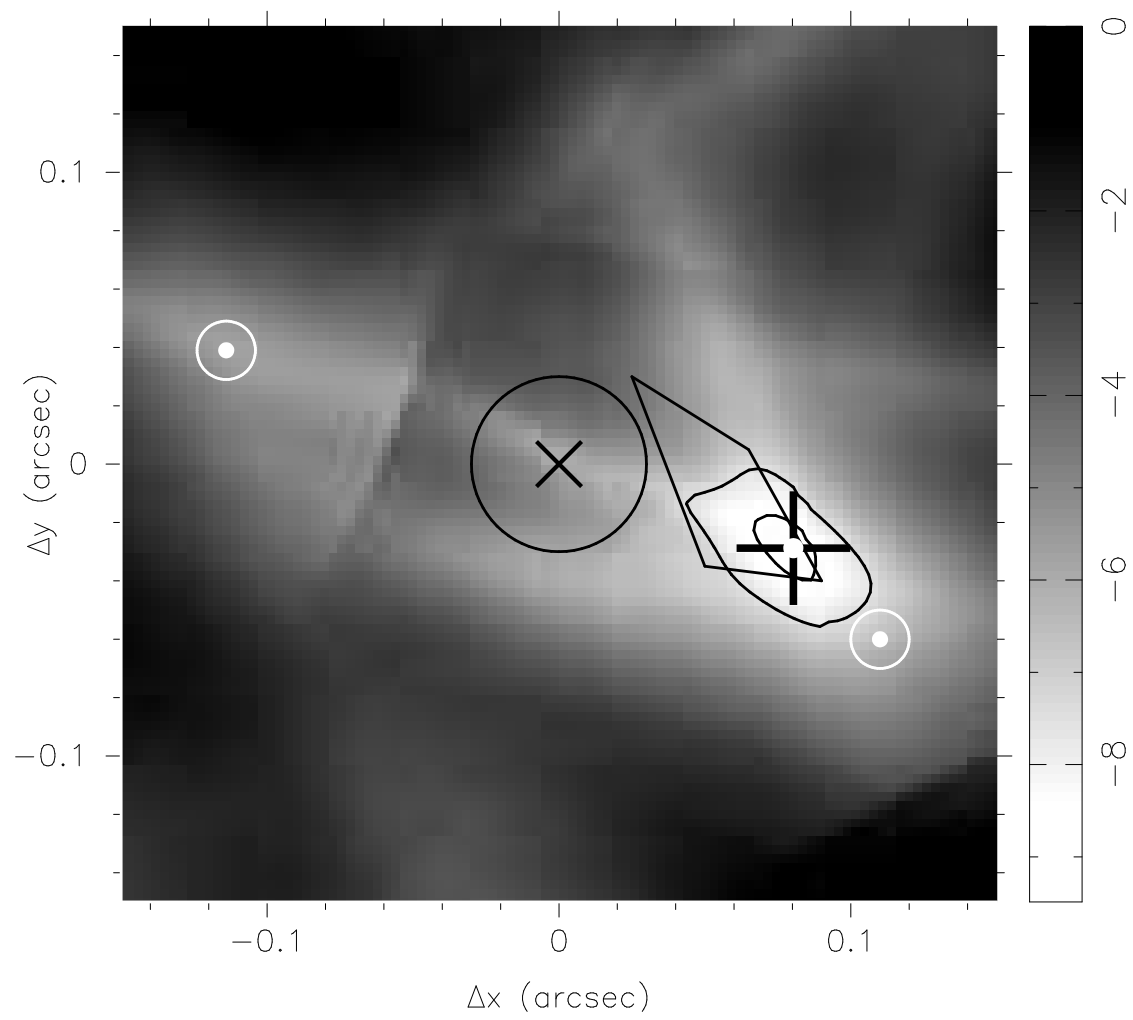

Figure 1.10. Pinpointing the $\mathrm{MBH}$ on the IR grid with gravitationally lensed stars (Alexander 2001). A gray scale plot of ln ML (shifted to 0 at the maximum) for 116 stars from the astrometric compilation by Genzel et al. (2000), as function of the shift in the astrometric grid over the central $0.3^{\prime \prime} \times 0.3^{\prime \prime}$ search field. The cross in the center is the origin according to the IR/radio alignment with its $1 \sigma$ error circle (Menten et al. 1997). The polygon is the $\sim 1 \sigma$ error region for the center of acceleration (Ghez et al. 2000). The circles are the observed IR sources with their 10 mas error circles. The most likely position of the $\mathrm{MBH}$ is indicated by a plus sign with $1 \sigma$ and $2 \sigma$ confidence level contours. (Reprinted with permission from The Astrophysical Journal).

result survives further scrutiny, it illustrates the potential of gravitational lensing as a tool for the study of the Galactic Center. This statistical method for locating the MBH by gravitational lensing should be re-applied whenever deeper astrometric data become available. 


\subsubsection{The detection of gravitational lensing}

The mode of detection of lensing events depends on the telescope's spatial resolution and its photometric sensitivity. When the two images can be resolved, as in the case discussed in $\S 1.4 .2$, the phenomenon is called a "macrolensing" event. When the two images cannot be resolved, only the variability in the flux of the lensed source is observed. This is called a "microlensing" event. Since $\theta_{E}$ increases with source distance behind the lens, there is a maximal source distance for microlensing, $D_{\mu}$, which can be estimated by noting that the angular distance between the two images close to peak magnification is $\sim 2 \theta_{E}$, and so

$$
D_{\mu}=\frac{D_{O L}}{\left(\theta_{\infty} / \phi\right)^{2}-1},
$$

where $\phi$ is the telescope's angular resolution, $\theta_{\infty} \equiv \sqrt{4 G M_{\bullet} / c^{2} D_{O L}} \sim$ $1.75^{\prime \prime}$ is the Einstein angle for a source at infinity, and it is assumed that $\phi<\theta_{E}$ is the criterion for resolving the two images.

The light curve for a constant velocity trajectory of a background source in the plane of the sky is given by substituting $y(t)$ in equation (1.21),

$$
y^{2}(t)=y_{0}^{2}+\mu^{2}\left(t-t_{0}\right)^{2},
$$

where $y_{0}$ is the impact parameter of the source trajectory relative to the lens, $\mu$ is the apparent motion, in units of $\theta_{E}$ per time, and $t_{0}$ is the time when $y=y_{0}$ (Figure 1.11). The resulting light curve is symmetric about $t_{0}$, and achromatic (i.e. has the same shape in every wavelength). If the photometric sensitivity is large enough to detect the unlensed source, the event will appear as a flaring up of a persistent source, otherwise, it will appear as a transient flare.

In order to plan the observational strategy for detecting gravitational lensing, or to estimate how likely it is that an observed flare is due to lensing, it is necessary to calculate the detection probability. Two quantities are commonly used to express this probability, the optical depth and the lensing rate. The optical depth for gravitational lensing is usually defined in relation to the probability of having at least one lens along the line of sight. If the cross-section of the lens at position $z_{i}$ is $S\left(z_{i}\right)$, and the number density of lenses there is $n\left(z_{i}\right)$, then the probability $P$ of having at least one lens along the line of sight is the complement of the probability of not encountering any lens,

$$
\begin{array}{rlrlll}
P & = & 1-\prod_{i}\left(1-n\left(z_{i}\right) S\left(z_{i}\right) \Delta z_{i}\right) & & \\
& = & 1-\exp \left(-\int_{0}^{z} n S d z^{\prime}\right) & & & \\
& = & 1-e^{-\tau} & \rightarrow & \tau & (\tau \ll 1)
\end{array}
$$




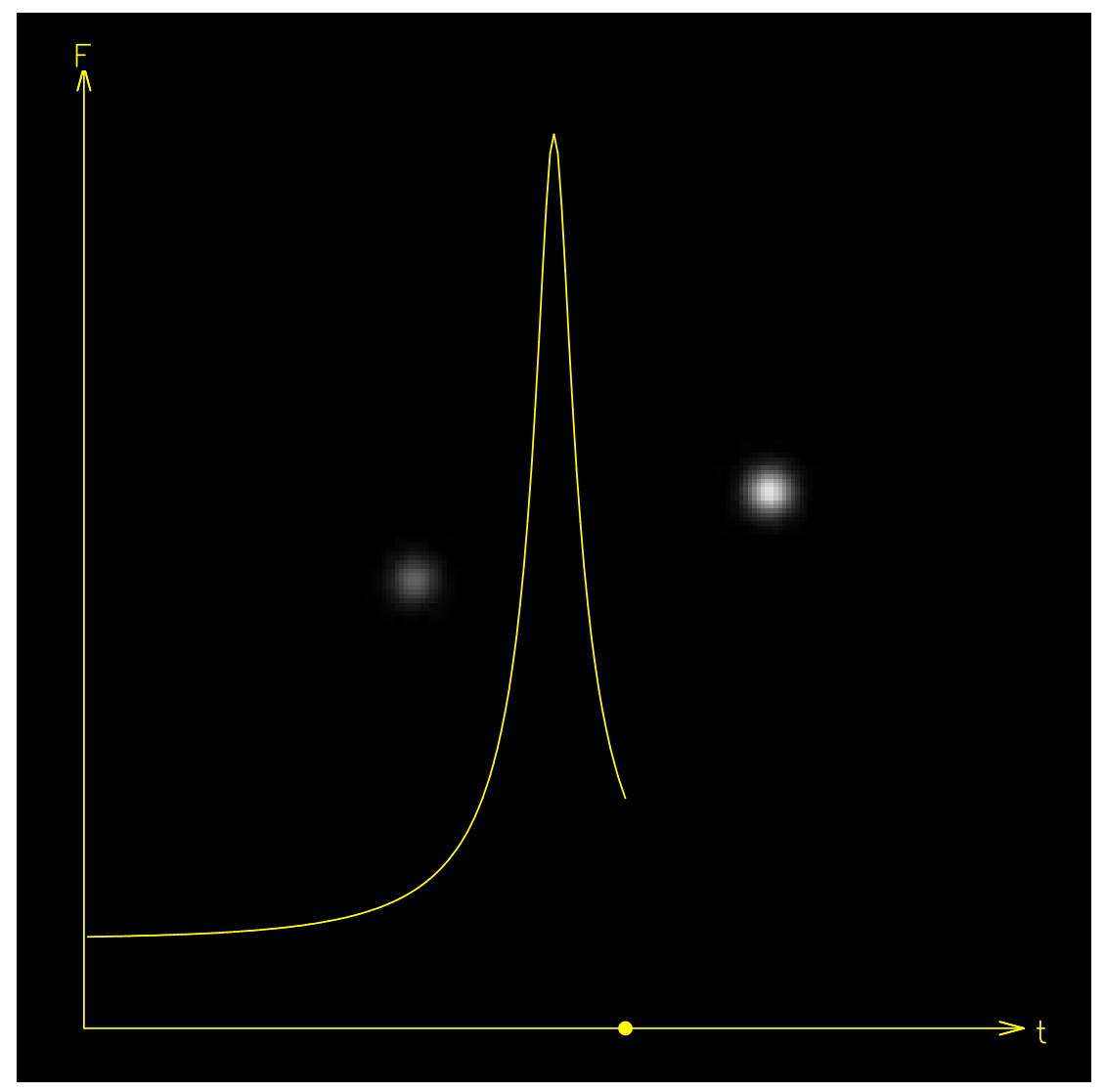

Figure 1.11. The microlensing light curve that corresponds to the lensing event in Figure 1.9 up to time $t=19$, in the case where the two images can not be resolved. The light curve is overlayed on the two lensed images as they would be observed if they could be resolved. The point on the time axis indicates the position of the source along its trajectory. The flux level on the left is the unmagnified flux of the source. The complete light curve will be symmetric relative to the peak flux.

It should be emphasized that $\tau$ is not a probability, and that $P$ and $\tau$ are interchangeable only when both are small. It is customary to define the lensing cross-section as $S=\pi \theta_{E}^{2}$, that is, the region where a source will be amplified by $A>1.34$ (equation 1.20). This definition is useful when there are many possible lines of sights, and it describes the probability that at any given instant a given line of sight will be lensed. This is relevant for Galactic microlensing searches, where millions of background 
stars are monitored simultaneously to find the rare one that is lensed by an intervening star. The observational situation for gravitational lensing by the $\mathrm{MBH}$ in the Galactic Center is different because the position of the lens is known and fixed, and so there is only one line of sight. In analogy to equation (1.26), the optical depth is defined in relation to the probability of having at least one source behind the lens along the line of sight,

$$
\tau=\int_{D_{O L}}^{\infty} n_{\star} \pi R_{E}^{2} d D_{O S}
$$

where $R_{E}$ is the physical size of $\theta_{E}$ at the source plane,

$$
R_{E}=\theta_{\infty} \sqrt{\left(D_{O S}-D_{O L}\right) D_{O S}} .
$$

Rough estimates predict $\tau \sim 1$ for lensing by the MBH (assuming no limits on the photometric sensitivity).

The optical depth does not take into account the relative motions of the lens and source, which reshuffle their random alignment and introduces a timescale to the problem. A more useful quantity for the lensing by the $\mathrm{MBH}$ is the lensing event rate with flux above a detection threshold $F_{0}$ due to the motion of sources behind the $\mathrm{MBH}$,

$$
\Gamma\left(>F_{0}\right) \simeq 2 \int_{D_{O L}}^{\infty} n_{\star} v \frac{R_{E}}{A} d D_{O S}, \quad A \geq \frac{F_{0}}{L_{\star} / 4 \pi D_{O S}^{2}}
$$

where $v_{\star}$ is the source star's projected velocity, $L_{\star}$ is its luminosity and $A \gg 1$ is assumed. For practical applications, equation (1.29) has to be modified to take into account the range of stellar luminosities and velocities, dust extinction, the total duration of the observations $T$ and the sampling rate $\Delta T$ (the mean duration of events amplified by more than $A$ is $\bar{t}=$ $\pi R_{E} / 2 A v$; only events with $\Delta T<t<T$ can be detected).

Figure 1.12 summarizes the dependence of the lensing cross-section, timescale and amplification on $D_{L S}$. The observational limitations, $F_{0}, T$ and $\Delta T$, place restrictions on $D_{L S}$ and the impact parameter for which sources can be detected, and affect the typical timescales and peak magnification that are likely to be observed. For example, high magnification events typically have longer time scales because the source trajectory must have a smaller impact parameter and so spends more time in the Einstein radius. Therefore, observations with limited temporal sampling will tend to pick out high magnification events.

Were any microlensing events detected? A couple of possible transient flaring events were detected very close to $\operatorname{Sgr} A^{\star}$ (Genzel et al. 1997; Ghez et al. 1998). For one of these a light curve was recorded, but as it was under-sampled only estimates of a timescale $(\sim 1 \mathrm{yr})$ and a typical magnification $(\sim A>5)$ could be derived from it. The a-posteriori probability of detecting a lensing event was estimated at only $0.5 \%$, but on 


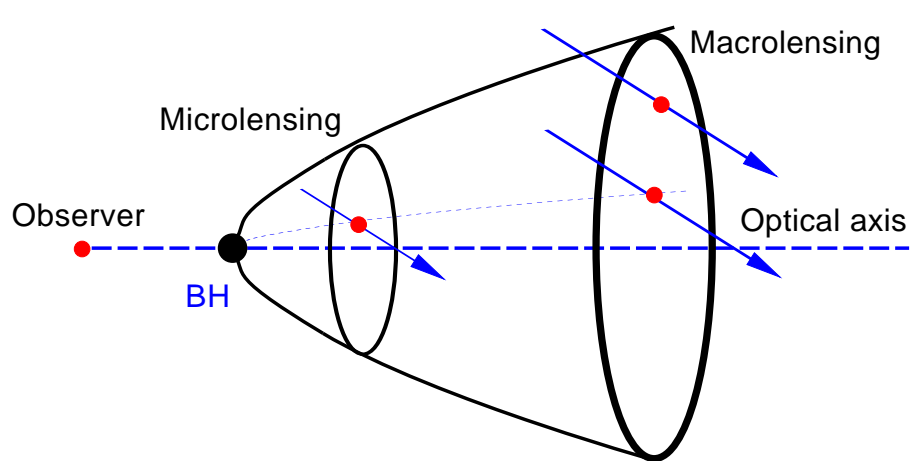

Figure 1.12. The lensing cross section, timescale and amplification as function of source distance behind the MBH. The size of the Einstein ring, or lensing cross-section, (cone, see equation 1.28) increases with distance behind the MBH. Close behind the MBH The Einstein ring (and the distance between the two images) is smaller than the telescope's resolution and the lensing appears as a microlensing event. Farther out, the Einstein ring is large enough for the two images to be resolved, and the lensing appears as a macrolensing event. The duration and peak magnification of the events depend on the impact parameter of the stellar trajectories (arrows). The closer they are to the optical axis, the longer the events and the higher the peak magnification. Trajectories with impact parameters at a fixed ratio of the Einstein radius (the two trajectories connected by the dotted line) will have the same peak amplification (equation 1.21), but the event duration will be longer for the sources farther away behind the MBH (assuming a uniform velocity field).

the other hand, the observed timescale and magnification are close to the median value that is expected for the observational limitations (Alexander \& Sternberg 1999). The interpretation of this event remains inconclusive.

\subsubsection{Magnification bias}

A lens magnifies by enlarging the angular size of the unlensed sky behind the lens, and since surface brightness is conserved, the fluxes of sources are magnified by the same amount. When the photometric sensitivity is such that all the stars can be detected even without being magnified, then the effect of lensing is to decrease the surface density of sources. However, if the fainter stars cannot be observed unless magnified, there are two possibilities (Figure 1.13): either there are enough faint sources that are magnified above the detection threshold to over-compensate for the decrease in surface 


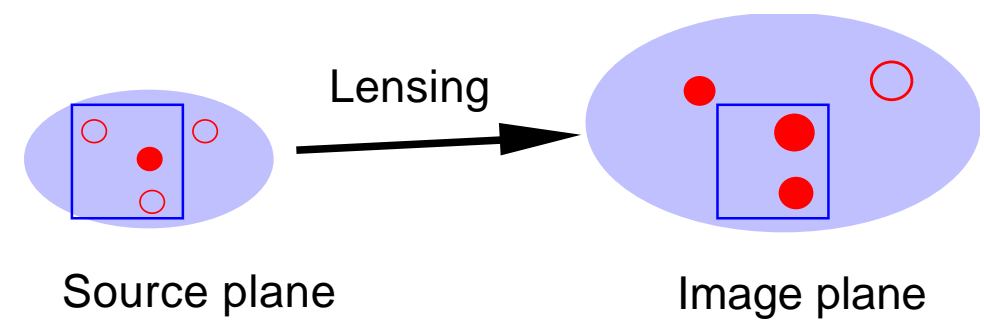

Figure 1.13. The magnification bias in star counts due to gravitational lensing. Stars (circles) are counted in a field of a fixed angular area (square) with a telescope of a given photometric detection threshold. The field in the unlensed sky (source plane, left), contains one bright star that can be observed (filled circle) and two stars that are too faint to be observed (open circles). Gravitational lensing stretches angular areas and amplifies fluxes by the same factor. The field in the lensed sky (image plane, right), now contains only two stars, but because they are magnified, both can be observed. This is an example of positive magnification bias, where gravitational lensing increases the apparent stellar surface density even as it decreases the total surface density. Negative magnification bias occurs when there aren't enough faint stars in the lensed population to compensate for the decrease in the total surface density.

density ("positive magnification bias"), or there aren't enough faint sources ("negative magnification bias"). The lensed luminosity function (number of stars per flux interval) is related to the unlensed one by

$$
\left.\left(\frac{d \Sigma}{d F}\right)_{\text {lensed }}\right|_{F}=\left.A^{-2}\left(\frac{d \Sigma}{d F}\right)_{\text {unlensed }}\right|_{F / A},
$$

where $\Sigma$ is the surface number density of stars and $F$ the flux. In many cases the luminosity function is well approximated by a power-law, $d \Sigma / d F \propto$ $F^{-\beta}$. It then follows from equation $(1.30)$ that for $\beta=2$ the decrease in the total surface density is exactly balanced by the magnification of faint stars above the detection threshold.

The chances for the detection of this effect in the Galactic Center appear small. A statistically meaningful detection requires a very high surface density that probably exceeds even that around the MBH (Wardle \& YusefZadeh 1992), and furthermore, models of the stellar luminosity function in the inner Galactic Center suggests that $\beta \sim 2$ for giants (Alexander \& Sternberg 1999). 


\subsubsection{Beyond the point mass lens approximation}

Up to this point we considered only the simple case of lensing by a point mass. There are two reasons to explore more complicated models. The first is that it would be useful if gravitational lensing could be used to dispel any remaining doubts that the dark compact mass in the Galactic Center is indeed a $\mathrm{MBH}$, and not some other extended distribution of matter, such as an compact cluster of stellar remnants (Maoz 1998) or a concentration of exotic particles (Tsiklauri \& Viollier 1998). Unfortunately, it can be shown the behavior of high-magnification light curves near peak magnification is universal and independent of the details of the lens (equation 11.21b of Schneider, Ehlers \& Falco 1992). For spherically symmetric mass distributions this implies that the light curves differ only in the low magnification tails, which are much harder to observe. The second reason is that the $\mathrm{MBH}$ is surrounded by a massive stellar cluster. Because the stellar mass is not smoothly distributed but is composed of discrete point masses, its effect on the lensing properties of the $\mathrm{MBH}$ is much larger than one may naively estimate by adding the stellar mass to that of the $\mathrm{MBH}$. We conclude the discussion of gravitational lensing in the Galactic Center with describing briefly the effect of enhanced lensing by stars near the $\mathrm{MBH}$ (Alexander \& Loeb 2001; Chanamé, Gould \& Miralda-Escudé 2001).

The effect of stars on lensing by the $\mathrm{MBH}$ is similar to that of planets on microlensing by a star, an issue that was studied extensively for the purpose of detecting planets by microlensing (e.g. Gould \& Loeb 1992). The lensing cross-section of an isolated star is $\theta_{E}^{2}\left(M_{\star}\right) / \theta_{E}^{2}\left(M_{\bullet}\right)=M_{\star} / M_{\bullet} \lesssim$ $10^{-6}$ smaller than that of the $\mathrm{MBH}$ (Equation 1.17). However, when the star lies near $\theta\left(M_{\bullet}\right)$, the shear of the MBH distorts its cross-section, which develops a complex topology, becomes radially elongated and is increased by up to an order of magnitude (Figure 1.14). As the stars orbit the MBH, their elongated cross-sections scan the lens plane. If these happen to intersect one of the images of a background source that is lensed by the MBH, the image will be split into 2 or 4 sub-images whose angular separation will be of order $\theta_{E}\left(M_{\star}\right)$, and so the sub-images will not be individually resolved. However, their combined flux will be significantly magnified. This will increase the probability of high magnification events over what is expected for lensing by the $\mathrm{MBH}$ alone. The light curves of such events will no longer be symmetric as they are for a point mass, but will exhibit a complex structure (e.g. Wambsganss 1997), and their typical variability timescales will rise sharply for images that lie near $\theta_{E}\left(M_{\bullet}\right)$ because of the increased stellar cross-section for lensing. Enhanced lensing by stars in the Galactic Center is estimated to increase the probability of $A>5$ lensing events by $\sim 2$ and of $A>50$ events by $\sim 3$. 


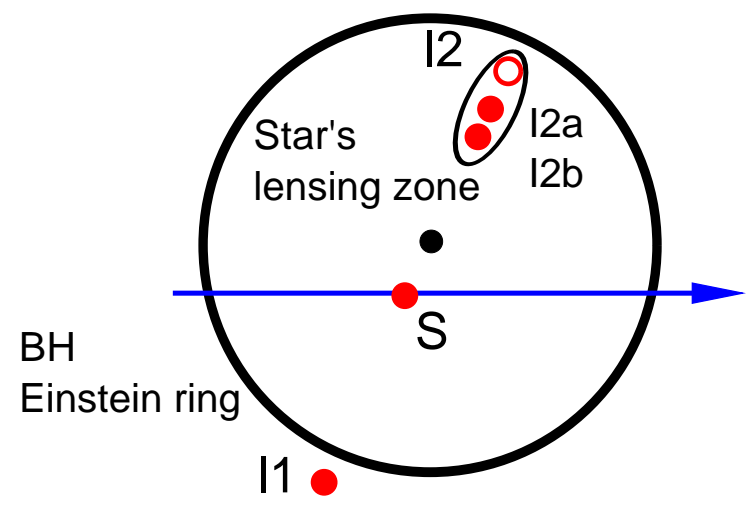

Figure 1.14. A schematic representation of lensing enhancement by a star near the MBH (Alexander \& Loeb 2001; Chanamé, Gould \& Miralda-Escudé 2001). A light source $\mathrm{S}$ passes behind the $\mathrm{MBH}$ (filled circle in the middle) and, in the absence of any other lensing mass, appears as two images: I1 outside the Einstein ring and $\mathrm{I} 2$ inside the Einstein ring. When one of the stars near the $\mathrm{MBH}$ happens to lie (in projection) close to I2, it will split I2 into two or four sub-images (here shown two), I2a and I2b. The star's Einstein ring is sheared by the potential of the $\mathrm{MBH}$ to an elongated shape of complex topology (represented here for simplicity as an ellipse), which increases in size the nearer I2 and the star are to the Einstein radius of the $\mathrm{MBH}$. This effect increases the cross-section for high magnification events above that of an isolated $\mathrm{MBH}$, and changes the character of the light curves.

\subsection{Summary}

Observations of the $\mathrm{MBH}$ in the Galactic Center present a unique opportunity to study the consequences of extreme stellar density, velocity and tidal fields on the dynamics and evolution of stars and their relation to the dynamics and evolution of the MBH. The existence of a high density relaxed stellar cusp around the $\mathrm{MBH}$ in the Galactic Center is theoretically motivated, and supported by observations. We explored some of the consequences of this environment for the appearance, internal structure and evolution of stars, through exotic object formation by direct collisions, collisional destruction of giant envelopes, stochastic tidal spin-up of stars by collisions with other stars, and extreme tidal interactions in the course tidal scattering by the MBH. It was shown that tidal processes have the potential of affecting a significant fraction of the stars over a large volume 
around the $\mathrm{MBH}$.

The MBH is also a gravitational lens. This can be used to probe the dark mass and the stars around it, but it also has the potential for complicating the interpretation of observations in the Galactic Center. Different detection modes were considered: macrolensing, microlensing, magnification bias, and the detection probability and detection rate were defined. Results from a statistical method for detecting lensed images and for pinpointing the $\mathrm{MBH}$ on the IR grid suggest that there may be a few far background supergiants that are lensed by the MBH. We described a lensing effect that involves both the $\mathrm{MBH}$ and the stars around it, and can increase the probability of high magnification events and modify the structure of the light curves.

The topics covered by this chapter by no means exhaust the scope of the subject. We did not address, among others, star formation near the $\mathrm{MBH}$, the role of stellar evolution in feeding the $\mathrm{MBH}$, or compact stellar remnants and x-ray sources. Some of these issues are discussed elsewhere in this book.

Over the next decade a wide array of IR instruments, both ground based and space borne, will improve the quality of photometric, spectroscopic and astrometric observations of the Galactic Center by orders of magnitude. Many of the issues discussed here will be resolved, as new questions will surely be raised. One thing is certain - we can look forward to exciting times in Galactic Center research.

\section{References}

Alexander, T., 1999, ApJ, 527, 835

Alexander, T., \& Sternberg A., 1999, ApJ, 520, 137

Alexander, T., 2001, ApJ, 553, L149

Alexander, T., \& Kumar P., 2001, ApJ, 549, 948

Alexander, T., \& Livio M., 2001, ApJ, 560, L143

Alexander, T., \& Loeb, A., 2001,, ApJ, 551, 223

Ayal, S., Livio, M., \& Piran, T., 2000, ApJ, 545, 772

Baganoff, F. K., et al., 2001, Nature, 413, 45

Bahcall, J., N., \& Wolf R., A., 1976, ApJ, 209, 214

Bahcall, J., N., \& Wolf R., A., 1977, ApJ, 216, 883

Binney, J., \& Tremaine S., 1987, Galactic Dynamics (Princeton: Princeton Univ. Press) p. 520

Blum, R. D., Sellgren, K., \& DePoy, D. L., 1996, ApJ, 470, 864

Chanamé, J., Gould, A., Miralda-Escudé, J., 2001, ApJ, 563, 793

Eckart, A., \& Genzel, R., 1997, MNRAS, 284, 576

Fragile, P. C., \& Mathews, G. J., 2000, ApJ, 542, 328

Frank, J., 1978, MNRAS, 184, 87

Frank, J. \& Rees, M. J., 1976, MNRAS, 176, 633 
Genzel, R., Eckart, A., Ott, T., \& Eisenhauer, F., 1997, MNRAS, 291, 219

Genzel, R., Hollenbach, D., \& Townes, C. H., 1994, Rep. Prog. Phys., 57, 417

Genzel, R., Pichon, C., Eckart, A., Gerhard, O. E., \& Ott, T., 2000, MNRAS, 317, 348

Genzel, R., Thatte, N., Krabbe, A., Kroker, H., \& Tacconi-Garman, L. E., 1996, ApJ, 472, 153

Ghez, A. M., Klein, B. L., Morris, M., \& Becklin, E. E. 1998, ApJ, 509, 678

Ghez, A. M., Morris, M., Becklin, E. E., Tanner, A. \& Kremenek T., 2000, Nature, 407, 349

Gould, A. \& Loeb, A., 1992, ApJ, 396, 104

Hills, J. G., 1975, Nature, 254, 295

Jaroszyński, M. 1998, Acta Astron., 48, 653

Jaroszyński, M. 1999, ApJ, 521, 591

Komossa, S., \& Bade, N., 1999, A\&A, 343, 775

Komossa, S., \& Greiner, J., 1999, A\&A, 349, 45

Lauer, T. R., Faber, S. M., Ajhar, E. A., Grillmair, C. J., \& Scowen, P. A., 1998, AJ, 116, 2263

Lightman, A. P., \& Shapiro, S., L., ApJ, 1977, 211, 244

Magorrian, J., \& Tremaine, S., 1999, MNRAS, 309, 447

Maoz, E., 1998, ApJ, 494, L181

Menten, K. M., Reid, M. J., Eckart, A., \& Genzel, R., 1997, ApJ, 475, L111

Monaghan, J. J., 1992, ARA\&A, 30, 543

Morris, M., 1993, ApJ, 408, 496

Murphy, B. W., Cohn, H. N., \& Durisen, R. H., 1991, ApJ, 370, 60

Press, W. H., \& Teukolsky, S. A., 1977, ApJ, 213, 183

Reid, M., 1993, ARA\&A, 31, 345

Renzini, A., Greggio, L., di Serego-Alighieri, S., Cappellari, M., Burstein, D., \& Bertola, F., 1995, Nature, 378, 39

Rubilar, G. F. \& Eckart, A., 2001, A\&A, 374, 95

Salim, S., \& Gould, A., 1999, ApJ, 523, 633

Schneider, P., Ehlers, J., \& Falco, E. E., 1992, Gravitational Lenses (New York: Springer)

Thorne, K. S., \& Zytkow, A. N., 1975, ApJ, 199, 19

Tsiklauri, D., \& Viollier, R. D., 1998, ApJ, 500, 591

Ulmer, A., Paczyński, B., \& Goodman, J., 1998, A\&A, 333, 379

Wambsganss, J., 1997, MNRAS, 284, 172

Wardle, M., \& Yusef-Zadeh, F., 1992, ApJ, 387, L65

Young, P., 1980, ApJ, 242, 1232 